\title{
Retinal oxygen metabolism in healthy subjects and glaucoma patients
}

\author{
Olof Birna Olafsdottir, ${ }^{1}$ Evelien Vandewalle, ${ }^{2,3}$ Luis Abegão Pinto, ${ }^{4}$ \\ Asbjorg Geirsdottir, ${ }^{1,5}$ Eline De Clerck, ${ }^{3}$ Peter Stalmans, ${ }^{3}$ Maria \\ Soffia Gottfredsdottir, ${ }^{5}$ Jona Valgerdur Kristjansdottir, ${ }^{5}$ Joachim Van Calster, ${ }^{3}$ \\ Thierry Zeyen, ${ }^{3}$ Einar Stefánsson, ${ }^{1,5}$ Ingeborg Stalmans ${ }^{2,3}$
}

\begin{abstract}
${ }^{1}$ Department of
Ophthalmology, University of Iceland, Reykjavik, Iceland

${ }^{2}$ Department of Ophthalmology Neurosciences, Laboratory of Ophthalmology, Katholieke Universiteit Leuven, Leuven, Belgium

${ }^{3}$ Department of Ophthalmology, University Hospitals Leuven, Leuven, Belgium

${ }^{4}$ Faculty of Medicine, Department of Pharmacology and Neurosciences, Lisbon University, Lisbon, Portugal ${ }^{5}$ Department of

Ophthalmology, Landspitali-The National University Hospital of Iceland, Reykjavik, Iceland
\end{abstract}

\section{Correspondence to}

Professor I Stalmans,

Department of Ophthalmology,

University Hospitals Leuven,

Campus St Raphaël,

Kapucijnenvoer 33, Leuven

B-3000, Belgium;

ingeborg.stalmans@uzleuven.be

$\mathrm{OBO}$ and $\mathrm{EV}$ contributed equally.

Received 24 January 2013 Revised 18 November 2013 Accepted 30 November 2013 Published Online First

8 January 2014

\section{ABSTRACT}

Background To test whether retinal oxygen metabolism is different in glaucoma patients compared with healthy subjects.

Methods This was a two-centre study where retinal vessel oxygen saturation was measured in glaucoma patients and healthy individuals with a non-invasive spectrophotometric retinal oximeter. Visual fields were obtained in the glaucoma patients.

Results No statistical difference was found in retinal oxygen saturation in arterioles $(p=0.16)$, venules $(p=0.16)$ and arteriovenous difference $(p=0.24)$ when all glaucoma patients $(n=74)$ were compared with healthy individuals $(n=89)$. When patients with advanced glaucoma (visual field mean defect (MD $\geq$ $10 \mathrm{~dB}, \mathrm{n}=21)$ ) were compared with healthy individuals, the oxygen saturation in venules was higher in glaucoma patients $(58.2 \% \pm 5.4 \%$ vs $53.8 \% \pm 6.4 \% ; p=0.0054$, mean \pm SD) and the arteriovenous difference was lower in glaucoma patients $(36.4 \% \pm 4.7 \%$ vs $39.5 \% \pm 5.7 \%$; $\mathrm{p}=0.021$ ). In glaucoma patients with mild glaucoma (visual field $M D \leq 5 d B, n=33$ ), no statistical differences were found in retinal oxygen saturation compared with healthy individuals.

Conclusions Glaucoma patients with advanced glaucoma have higher oxygen saturation in venules and lower arteriovenous difference in oxygen saturation compared with healthy individuals. The decreased arteriovenous difference in severe glaucoma may be related to lower oxygen consumption secondary to neuropathy.

\section{INTRODUCTION}

Glaucoma is a chronic progressive optic neuropathy with corresponding visual field defects and structural changes at the optic nerve. The exact cause of glaucoma is unknown, but there are indications that vascular abnormalities might be present. ${ }^{1-3}$ There is, however, limited knowledge about metabolic alterations in the retina of glaucomatous subjects.

We recently reported abnormalities in retinal oxygen metabolism in patients with advanced glaucomatous visual field defects, ${ }^{4}$ and with decreasing neuroretinal rim area of the optic disc as well as nerve fibre layer thinning. ${ }^{5}$ These studies were performed with non-invasive spectrophotometric retinal oximetry ${ }^{6} 7$ and indicated increased haemoglobin oxygen saturation in retinal venules in glaucomatous eyes with advanced visual field defects compared with those with mild visual defects. We now expand this investigation and compare retinal oxygen saturation in patients with open-angle glaucoma to a healthy population.

The aim of the study is to find whether oxygen saturation in retinal blood vessels is different in healthy subjects and patients with glaucoma and specifically in glaucoma with mild or advanced visual field defects.

\section{MATERIALS AND METHODS}

This was a two-centre study where data were collected at the Department of Ophthalmology in the University Hospitals Leuven, Leuven, Belgium, and at the Department of Ophthalmology, Landspitali-The National University Hospital of Iceland, Reykjavik, Iceland.

The study was a prospective, non-randomised clinical trial. It was approved by the Institutional Review Board of the University Hospitals Leuven, the Icelandic National Bioethics Committee and Data Protection Authority. The study adhered to the tenets of the Declaration of Helsinki. All eligible patients who agreed to participate in the study signed an informed consent prior to enrolment.

The same standard protocol was followed in both centres. To maintain comparability of the data, an experienced researcher from the Icelandic group trained the researchers in Belgium. In Belgium, patients were recruited at the glaucoma division of the Department of Ophthalmology at the University Hospitals Leuven. In Iceland, patients diagnosed with glaucoma were recruited from a glaucoma clinic at the Landspitali-The National University Hospital of Iceland and from a private clinic (Augnlæknar Reykjavíkur, Hamrahlid 17, Reykjavik).

Included in the study were healthy individuals and glaucoma patients with no other ocular disease. All participants were of age 40 years or older. Exclusion criteria consisted of any other ocular diseases than open-angle glaucoma and systemic diseases such as diabetes. Individuals receiving antihypertensive medication for elevated systemic blood pressure as well as individuals with mild cataract were not excluded due to the prevalence of these conditions in the age group that was studied.

In all glaucoma patients, the diagnosis had been made by glaucoma specialists (IS, MSG). Glaucoma was defined as having characteristic optic disc damage (based on cup/disc ratio, thinning of neuroretinal rim, notching, disc haemorrhages, etc.) and by corresponding visual field defects. For the 
diagnosis of primary open-angle glaucoma (POAG), an untreated intraocular pressure (IOP) of $>21 \mathrm{~mm} \mathrm{Hg}$ was required, while patients with IOPs consistently $\leq 21 \mathrm{~mm} \mathrm{Hg}$ were classified as having normal tension glaucoma (NTG). In each patient, the eye with the worse visual field damage was included in the study. Consecutive glaucoma patients in each glaucoma clinic that did not meet the exclusion criteria were invited to join the study. Seventy-four glaucoma patients were recruited; 15 from Iceland and 59 from Belgium. In Belgium, healthy individuals were recruited from the general consultation at the same department. In Iceland, healthy individuals were recruited through an advertisement. Every healthy individual was examined by an ophthalmologist (IS, AG) to exclude glaucoma and other diseases. In the healthy population, both eyes were studied but only one eye was randomly chosen with the Microsoft Excel rand function. Overall, 89 healthy individuals were recruited; 72 from Iceland and 17 from Belgium. The characteristics of the study groups are described in table 1 .

Non-invasive spectrophotometric retinal oximetry was performed on the same day as the ophthalmological evaluations. The Oxymap Retinal Oximeter, Oxymap T1 (Oxymap ehf, Reykjavik, Iceland), consists of a fundus camera (Topcon TRC-50DX, Topcon Corporation, Tokyo, Japan) with an attached image splitter and two digital cameras (Insight IN1800, Diagnostic Instruments Inc, Michigan, USA). The instrument (described in detail elsewhere ${ }^{7}$ ) provides two images simultaneously at two different wavelengths, $600 \mathrm{~nm}$, which is sensitive to oxygen saturation, and $570 \mathrm{~nm}$, which is not sensitive to oxygen saturation. The optical density (OD) of retinal vessels from the two acquired images is automatically calculated by software algorithm according to the equation $\mathrm{OD}=\log \left(\mathrm{I}_{0} / \mathrm{I}\right)$, where $I_{0}$ is light reflected by the background to the side of the vessel and $I$ is the light reflected from the vessel. The ratio of the $\mathrm{OD}$ at $600 \mathrm{~nm}$ and $\mathrm{OD}$ at $570 \mathrm{~nm}$ is inversely related to haemoglobin oxygen saturation. ${ }^{8} 9$

Relative oxygen saturation was measured automatically with the Oxymap Analyzer Software 2.2.1 (v.3847) in selected vessel segments in the pseudocolour fundus image the software

Table 1 Baseline characteristics of healthy subjects and glaucoma patients

\begin{tabular}{|c|c|c|c|}
\hline & Healthy & Glaucoma & $\begin{array}{l}p \\
\text { Value }\end{array}$ \\
\hline Number of patients & 89 & 74 & - \\
\hline \multicolumn{4}{|l|}{ Gender } \\
\hline Females & 47 & 40 & - \\
\hline Males & 32 & 34 & - \\
\hline \multicolumn{4}{|l|}{ Numbers of eyes } \\
\hline Right eyes & 42 & 33 & - \\
\hline Left eyes & 47 & 41 & - \\
\hline Age, mean $\pm S D$, years & $58 \pm 10$ & $64 \pm 12$ & 0.0007 \\
\hline Intraocular pressure, mean $\pm \mathrm{SD}(\mathrm{mm} \mathrm{Hg})$ & $15 \pm 3$ & $15 \pm 4$ & 0.31 \\
\hline Heart rate, mean \pm SD $(\mathrm{bpm})$ & $70 \pm 14$ & $65 \pm 12$ & 0.025 \\
\hline Systolic blood pressure, mean $\pm \mathrm{SD}(\mathrm{mm} \mathrm{Hg})$ & $135 \pm 20$ & $147 \pm 12$ & 0.0021 \\
\hline Diastolic blood pressure, mean $\pm \mathrm{SD}(\mathrm{mm} \mathrm{Hg})$ & $85 \pm 11$ & $85 \pm 11$ & 0.79 \\
\hline Mean arterial pressure, mean \pm SD $(\mathrm{mm} \mathrm{Hg})$ & $102 \pm 12$ & $106 \pm 13$ & 0.079 \\
\hline $\begin{array}{l}\text { Retinal perfusion pressure, mean } \pm \text { SD } \\
(\mathrm{mm} \mathrm{Hg})\end{array}$ & $53 \pm 8$ & $56 \pm 9$ & 0.086 \\
\hline Number of glaucoma medications, mean $\pm S D$ & - & $1.9 \pm 1.4$ & - \\
\hline Trabeculectomy & - & 20 & - \\
\hline Shunt (Baerveldt) & - & 1 & - \\
\hline
\end{tabular}

makes from the two images (figure 1). Retinal oxygen saturation was measured in the first-degree vessels, or second degree if the length of the first degree was less than 50 pixels. Both retinal arterioles and venules inferior and superior to the optic nerve head were measured. Only retinal vessels with a width of more than six pixels, which equals approximately 56 micrometers, ${ }^{10}$ and a length between 50 and 200 pixels were analysed. Around the optic disc, an area of 15 pixels as well as branching vessels and their origin were manually excluded. Because there is an artifactual decrease in measured saturation values with increased vessel diameter in both retinal arterioles and venules, ${ }^{11}$ all measurements were manually corrected by adding $1.16 \%$ to the saturation value for each pixel above the mean diameter for arterioles and venules. Also, $1.16 \%$ was subtracted for each pixel below mean diameter. The correction was determined by comparing saturation just before and just after bifurcation of vessels where the vessel diameter is different but the saturation can be assumed to be the same. ${ }^{7}$ The vessel diameter measurements with the oximeter are consistent and repeatable. ${ }^{10}$

The arteriovenous difference was calculated by oxygen saturation in venules subtracted from oxygen saturation in arterioles.

All measurements were performed in darkness, after dark adaptation of the subjects for around $2 \mathrm{~min}$. At least two images with the optic disc in the centre of the image were acquired from each individual. The time between images (flashes) was about $30 \mathrm{~s}$ on average. Retinal oxygen saturation was measured in the best-quality image that was acquired.

In Belgium, IOP was measured using a Goldmann applanation tonometry mounted on a Haag-Streit slit lamp (Haag-Streit BQ 900, Haag-Streit International, Köniz, Switzerland). The eyes were anesthetised with oxybuprocaine $0.4 \%$ (Unicaïne $0.4 \%$, Théa Pharma, Wetteren, Belgium). In Iceland, IOP was measured with iCare TAO1 tonometer (Tiolat Oy, Helsinki, Finland). Comparisons have shown that IOP readings with iCare are clinically reproducible ${ }^{12}$ and in agreement with those obtained by Goldmann applanation tonometry. ${ }^{13-15}$

Pupils were dilated with tropicamide $0.5 \%$ (Tropicol, Théa Pharma, Wetteren, Belgium) and phenylephrine 5\% (Neosynephrin-POS, Ursapharm, Saarbrücken, Germany) in Belgium and with 1\% tropicamide (Mydriacyl, S.A. AlconCouvreur N.V., Puurs, Belgium) in Iceland, which in some cases was supplemented with $10 \%$ phenylephrine hydrochloride (AK-Dilate, Akorn Inc, Lake Forest, Illinois, USA). Both dilation protocols were checked, and dilation by the addition of phenylephrine to tropicamide did not influence the retinal oxygen saturation values or the vessel diameter. ${ }^{16}$

In Belgium, the automated perimetry was performed using a Humphrey Field Analyzer, program 24-2, Sita standard strategy (Carl Zeiss, Oberkochen, Germany) or Octopus 301, program G1 $\left(30^{\circ}\right)$, dynamic strategy (Interzeag AG, Schlieren, Switzerland). In Iceland, visual field testing was performed using an Octopus 123 (Interzeag AG, Schlieren, Switzerland), also using program G1. Unreliable visual fields (false positive, false negative or fixation loss values $>20 \%$ ) were excluded. Visual fields with a mean defect (MD) $\leq 5 \mathrm{~dB}$ were defined as 'mild glaucoma', and visual fields with a $\mathrm{MD} \geq 10 \mathrm{~dB}$ were defined as 'advanced glaucoma'. The mean deviation of the Humphrey perimeter was converted to MD usable with the Octopus perimeter with the PeriData analyse software, V.2.3.

In both countries, systolic and diastolic blood pressures (SP and DP, respectively) were measured using an automatic sphygmomanometer (in Belgium: Omron HEM-7001-E, Omron, Kyoto, Japan; in Iceland: Omron HEM-7221-E, Omron, Kyoto, 

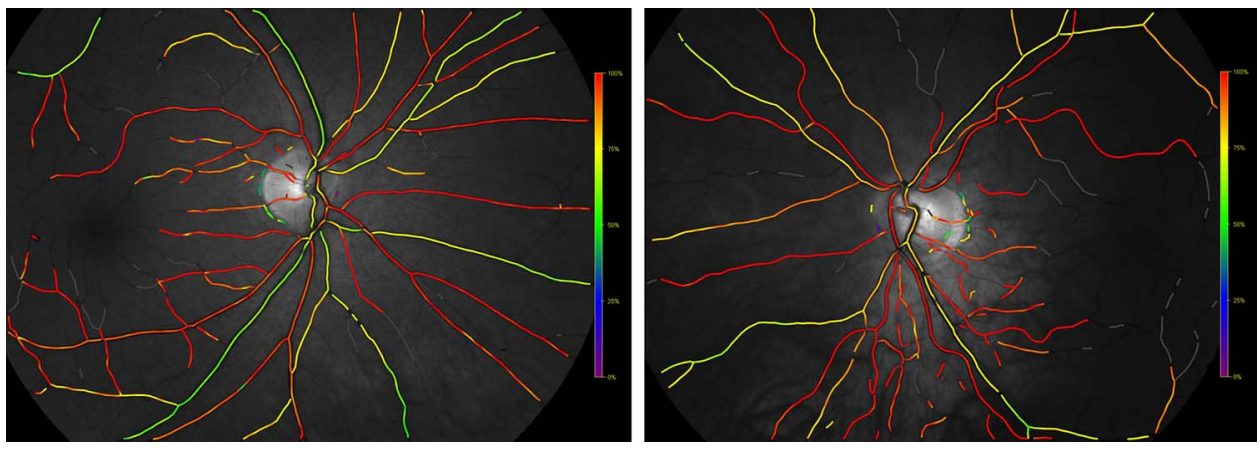

Figure 1 Pseudocolour fundus map showing oxygen saturation in a healthy individual (left) and a glaucoma patient (right). The difference in oxygen saturation in the venules is visible where in the healthy individual the venules are of green colour, which denotes oxygen saturation around $50 \%$, and in the advanced glaucoma patient the venules are of yellow colour, which denotes oxygen saturation around $60-65 \%$.

Japan) after a $5 \mathrm{~min}$ resting period. Mean arterial pressure (MAP) was calculated as $\mathrm{MAP}=2 / 3 \mathrm{DP}+1 / 3 \mathrm{SP}$. Mean ocular perfusion pressure was calculated as 2/3MAP-IOP.

The number of ocular hypotensive medications was also recorded. The fixed combinations were documented according to the number of active ingredients. Systemic carbonic anhydrase inhibitors were counted as one medication.

Statistical analysis was performed with Prism, V.5 (Graphpad Software Inc, La Jolla, California, USA). Normal distribution of the data was verified with the D'Agostino and Pearson omnibus normality test. If the data were normally distributed, a twotailed, unpaired Student $t$ test was used for the comparison of the two groups. Otherwise, the non-parametric Mann-Whitney $\mathrm{U}$ test was applied. $\mathrm{p}<0.05$ was considered statistically significant.

\section{RESULTS}

No statistical difference was found in retinal oxygen saturation in arterioles when all glaucoma patients $(n=74)$ were compared with healthy individuals $(\mathrm{n}=89, \mathrm{p}=0.16$, table 2$)$. There was also no statistical difference in oxygen saturation in venules in all glaucoma patients compared with healthy individuals $(p=0.16)$ as well as in arteriovenous difference $(p=0.24$; table 2 and figure 2).
In patients with mild glaucoma (visual field $M D \leq 5 \mathrm{~dB}, \mathrm{n}=33$ ) compared with healthy individuals, no statistical difference was found in retinal oxygen saturation in arterioles $(p=0.26)$, venules $(p=0.87)$ or arteriovenous difference $(p=0.75$; table 2 and figure 2). In patients with advanced glaucoma (visual field $M D \geq 10 \mathrm{~dB}, \mathrm{n}=21$ ) versus healthy individuals, there was no difference in arteriole oxygen saturation $(p=0.19)$. However, the oxygen saturation in venules was higher in glaucoma patients with advanced visual field defects compared with healthy individuals $(58.2 \% \pm 5.4 \%$ vs $53.8 \% \pm 6.4 \%$; mean $\pm S D, p=0.0054$; figure 2 and table 2). The arteriovenous difference was lower in glaucoma patients with advanced visual field defect compared with healthy individuals $(36.4 \% \pm 4.7 \%$ vs $39.5 \% \pm 5.7 \%$, $\mathrm{p}=0.021$; table 2 and figure 2 ).

When glaucoma patients with advanced glaucoma were compared with patients with mild glaucoma, no statistical difference was found in oxygen saturation in arterioles $(p=0.68$; table 2$)$. The oxygen saturation in venules was higher in patients with advanced glaucoma compared with patients with mild glaucoma $(58.2 \% \pm 5.4 \%$ vs $53.8 \% \pm 7.6 \%, \mathrm{p}=0.026$; table 2 and figure 2$)$, and the arteriovenous difference was lower in patients with advanced glaucoma compared with patients with mild glaucoma (36.4\% $\pm 4.7 \%$ vs $40.4 \% \pm 7.0 \%, p=0.035$; table 2 and figure 2 ).

No differences were observed between POAG and NTG patients, independent of the severity of the visual field defects.

Table 2 Mean oxygen saturation in healthy subjects and glaucoma patients

\begin{tabular}{|c|c|c|c|c|}
\hline & \multicolumn{4}{|c|}{ Oxygen saturation $(\%$, mean \pm SD) } \\
\hline & \multirow[b]{2}{*}{ Healthy $(n=89)$} & \multicolumn{3}{|l|}{ Glaucoma } \\
\hline & & All glaucoma $(n=74)$ & Mild glaucoma ( $n=33$ ) & Advanced glaucoma ( $n=21)$ \\
\hline Arterioles & $93.3 \pm 4.1$ & $94.1 \pm 3.1$ & $94.2 \pm 3.2$ & $94.5 \pm 2.4$ \\
\hline Venules & $53.8 \pm 6.4$ & $55.5 \pm 7.1$ & $53.8 \pm 7.6$ & $58.2 \pm 5.4$ \\
\hline \multirow[t]{3}{*}{ AV difference } & $39.5 \pm 5.7$ & $38.5 \pm 6.5$ & $40.4 \pm 7.0$ & $36.4 \pm 4.7$ \\
\hline & \multicolumn{4}{|l|}{$p$ Values } \\
\hline & Healthy versus all glaucoma & Healthy versus mild glaucoma & Healthy versus advanced glaucoma & Mild glaucoma versus advanced glaucoma \\
\hline Arterioles & 0.16 & 0.26 & 0.19 & 0.68 \\
\hline Venules & 0.16 & 0.87 & 0.0054 & 0.026 \\
\hline AV difference & 0.24 & 0.75 & 0.021 & 0.035 \\
\hline
\end{tabular}



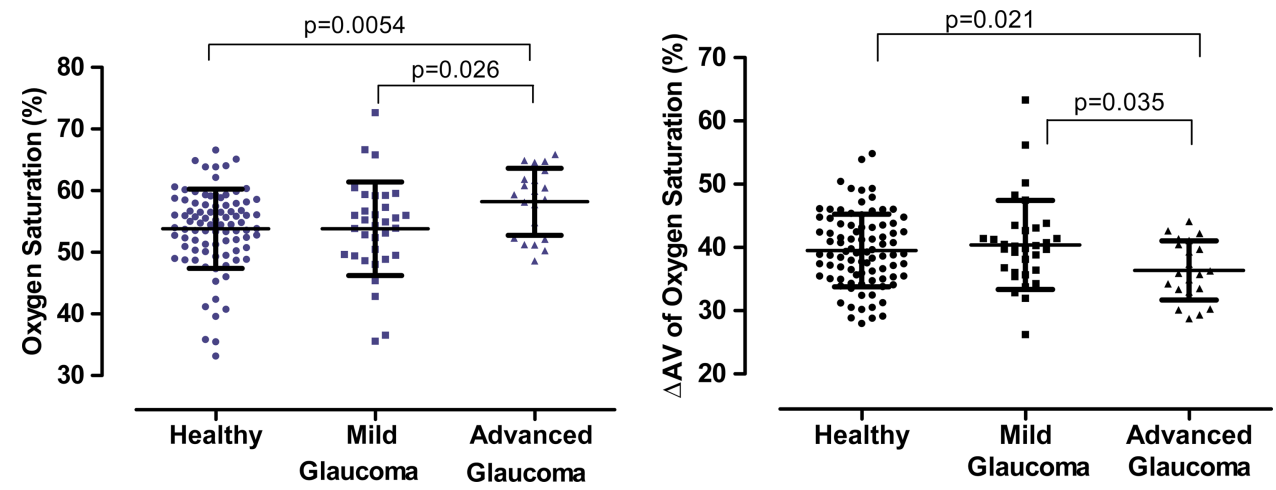

Figure 2 Left: Retinal oxygen saturation in venules was higher in glaucoma patients with poor visual field ( $n=21$, visual field mean defect $(M D) \geq 10 d B)$ compared with healthy individuals $(n=89, p=0.0054)$ and with patients with mild glaucoma (visual field $M D \leq 5 d B, n=33, p=0.026)$. Right: Retinal oxygen saturation arteriovenous (AV) difference was lower in patients with advanced glaucoma compared with healthy individuals $(p=0.021)$ and with patients with mild glaucoma $(p=0.035)$.

\section{DISCUSSION}

There was no difference in retinal oxygen saturation between healthy individuals and the total group of glaucoma patients. However, patients with advanced glaucoma had higher oxygen saturation in the venules and lower arteriovenous difference compared with healthy individuals and also patients with mild glaucoma.

Our data indicate that oxygen extraction is lower in patients with advanced glaucomatous visual field defects compared with healthy individuals and patients with mild glaucoma. Higher oxygen levels in venules and smaller arteriovenous difference in advanced glaucoma point to less oxygen demand of the tissue. This finding may result from tissue atrophy and therefore decreased oxygen consumption of the tissue. The observation of abnormal oxygen metabolism in advanced glaucoma but not in mild glaucoma suggests that this abnormality is more likely secondary to glaucomatous atrophy than a primary cause of the disease. In primary ischaemia, where blood flow is insufficient for the oxygen demand of the tissue, it would be expected that the tissue should extract an increased proportion of oxygen from the blood, and venular oxygen saturation would fall, which we, however, did not measure.

If ischaemia were the primary cause of glaucoma, we would expect to see hypoxia as a consequence of ischaemia. This is not what we observed in the study, considering that the oxygen saturation in venules was increased in advanced glaucoma. However, these patients were all under active ophthalmological care and with reasonably normal IOPs. It is possible that abnormalities in retinal oxygen metabolism in patients with early glaucoma may be intermittent, for example, when IOP is high or nocturnal blood pressure is low. Those ocular perfusion pressure dips could lead to intermittent ischaemia. Therefore, while our data do not support the ischaemia/hypoxia hypothesis for glaucoma pathophysiology, they do not completely reject it. Further oximetry studies on patients with very high IOP, low-ocular perfusion pressure, patients with rapidly progressive glaucoma and various types of glaucoma are needed to provide further testing of the ischaemia/hypoxia hypothesis. A longitudinal, prospective study is also mandatory to test this hypothesis. Also, it would be interesting to measure blood flow in the same group of patients so that oxygen delivery, the product of blood flow and arteriovenous difference in oxygen content could be calculated. Finally, the retinal circulation is not the only possible culprit; optic nerve hypoxia may exist independently of retinal oxygen metabolism.
Other studies have been performed on glaucoma and retinal oxygen saturation. Michelson and Scibor measured the retinal oxygen saturation in high-tension and low-tension glaucoma patients that were similar to our mild glaucoma group and in healthy subjects. ${ }^{17}$ Our data confirm their previous findings where they found no differences in oxygen saturation in venules or oxygen extraction between the three groups. They found a significant correlation between the neuroretinal rim area and decreased arteriovenous differences, which suggests reduced metabolism due to tissue loss. This has been confirmed by our previous publication ${ }^{5}$ and is in agreement with our measurements on functional (visual field) damage. However, they found that the arterial oxygen saturation was significantly lower in NTG compared with healthy subjects, which could not be confirmed in our study.

Ito $e t a l^{18}$ also measured retinal oxygen saturation in hightension and low-tension glaucoma patients and healthy individuals. They found that the average oxygen saturation of five juxtapapillary points was significantly higher in healthy individuals compared with glaucoma patients. In the superior and nasal quadrant, they found that the low-tension glaucoma had lower oxygen saturations compared with the high-tension glaucoma. A limitation of this study was that two-dimensional resolution was not precise enough to measure oxygen saturation of a defined point or area due to the large pixel size in their system. Moreover, they experienced problems with eye movements, which is not a factor in our oximetry system because the two images used to calculate the oxygen saturation were taken simultaneously by the oximeter. Probably the most important limitation for comparison with our results is that the signal for measurements in their oximeter is different, that is, from the capillaries. It may be difficult to differentiate the signal from the capillaries from the choroid.

Subgroup analysis in the Icelandic group as well as in the Belgium group confirmed the non-difference between healthy individuals and glaucoma patients. However, the number of patients with advanced glaucoma in the Icelandic group was too small to perform pairwise comparison with healthy individuals and between the subgroups. The oxygen saturation in healthy individuals was slightly higher in Belgium than in Iceland, and therefore the arteriovenous difference was slightly lower in Belgium, but other measurements compared well.

Our study has some limitations. Patients were taking different glaucoma medications, and some of our patients had already undergone glaucoma surgery. Some medications can have an 
effect on the retinal oxygen saturation by influencing the retinal blood flow. ${ }^{19}{ }^{20}$ Hardarson et $a l^{21}$ found a $2 \%$ oxygen increase in oxygen saturation in arterioles after trabeculectomy. There was also a significant age difference between glaucoma patients and healthy individuals. Therefore, oxygen saturation values should be cautiously interpreted. However, our high number of somewhat heterogeneous study population is representative for a real-time glaucoma patient population, and our results provide proof of concept in this non-selected two-centre patient group.

In conclusion, glaucoma patients with advanced visual fields have reduced oxygen extraction compared with healthy controls, possibly related to tissue atrophy and perhaps reduced consumption. No difference is found between healthy controls and patients with mild glaucoma. Retinal oximetry offers a tool to further test the role of oxygen metabolism, ischaemia and hypoxia in glaucoma in a prospective trial.

Acknowledgements The authors would like to thank Sveinn Hakon Hardarson, Sien Boons and Veerle Vanbellinghen for their technical support.

Contributors All authors contributed to the study in such manner that their names should appear on the article. OBO, EV, ES and IS were responsible for the design and conduct of the study. All authors were responsible for collection, management, analysis and interpretation of the data, preparation, review and approval of the article.

Funding EV was supported with a grant from the FWO 'Fonds Wetenschappelijk Onderzoek-Vlaanderen'. OBO was supported with a grant from the Icelandic Centre for Research (Rannis no. 100429021). This work was supported by an unrestricted grant from Merck Sharp and Dohme (MSD), Prevention of Blindness Fund and Landspitali-University Hospital Research Fund (no. A-2013-023). The funding source had no involvement in the study.

Competing interests There are no relationships, conditions or circumstances that present potential conflict of interest except for ES. ES is involved in the development of the instrument (oximeter) used and has a financial interest in the company Oxymap ehf., which makes the instrument.

Ethics approval The Institutional Review Board of the University Hospitals Leuven, the Icelandic National Bioethics Committee and Data Protection Authority (Iceland).

Provenance and peer review Not commissioned; externally peer reviewed.

Data sharing statement Additional data are available upon request from the corresponding author, IS, as well as ES.

\section{REFERENCES}

1 Flammer J. The vascular concept of glaucoma. Surv Ophthalmol 1994;38(Suppl): S3-6.

2 Flammer J, Orgul S, Costa VP, et al. The impact of ocular blood flow in glaucoma. Prog Retin Eye Res 2002;21:359-93.
3 Pournaras CJ, Rungger-Brandle E, Riva CE, et al. Regulation of retinal blood flow in health and disease. Prog Retin Eye Res 2008;27:284-330.

4 Olafsdottir OB, Hardarson $\mathrm{SH}$, Gottfredsdottir MS, et al. Retinal oximetry in primary open-angle glaucoma. Invest Ophthalmol Vis Sci 2011;52:6409-13.

5 Vandewalle $E$, Pinto LA, Olafsdottir OB, et al. Oximetry in glaucoma: correlation of metabolic change with structural and functional damage. Acta Ophthalmol 2013. Published Online First: 17 January 2013. doi:10.1111/aos.12011

6 Hardarson $\mathrm{SH}$, Harris A, Karlsson RA, et al. Automatic retinal oximetry. Invest Ophthalmol Vis Sci 2006;47:5011-16.

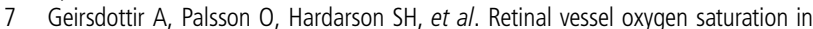
healthy individuals. Invest Ophthalmol Vis Sci 2012;53:5433-42.

8 Harris A, Dinn R, Kagemann L, et al. A review of methods for human retinal oximetry. Ophthalmic Surg Lasers Imaging 2003;34:152-64.

9 Beach J, Schwenzer K, Srinivas S, et al. Oximetry of retinal vessels by dual-wavelength imaging: calibration and influence of pigmentation. J Appl Physiol 1999;86:748-58

10 Blondal R, Sturludottir MK, Hardarson SH, et al. Reliability of vessel diameter measurements with a retinal oximeter. Graefes Arch Clin Exp Ophthalmol 2011;249:1311-17.

11 Gottfredsdottir MS, Bergvinsson HR, Blondal R, et al. Retinal oxygen saturation measurements: repeatability and influence of vessel diameter. ARVO Meeting Abstracts 2011;52:5668.

12 Salvetat ML, Zeppieri M, Miani F, et al. Comparison of iCare tonometer and Goldmann applanation tonometry in normal corneas and in eyes with automated lamellar and penetrating keratoplasty. Eye (Lond) 2011;25:642-50.

13 Brusini P, Salvetat ML, Zeppieri M, et al. Comparison of ICare tonometer with Goldmann applanation tonometer in glaucoma patients. J Glaucoma 2006:15:213-17.

14 Scuderi GL, Cascone NC, Regine F, et al. Validity and limits of the rebound tonometer (ICare(R)): clinical study. Eur J Ophthalmol 2011;21:251-7.

15 Vandewalle E, Vandenbroeck S, Stalmans I, et al. Comparison of ICare, dynamic contour tonometer, and ocular response analyzer with Goldmann applanation tonometer in patients with glaucoma. Eur J Ophthalmol 2009;19:783-9.

16 Vandewalle $\mathrm{E}$, Abegao Pinto L, Olafsdottir OB, et al. Phenylephrine $5 \%$ added to Tropicamide $0.5 \%$ eye drops does not influence retinal oxygen saturation values or retinal vessel diameter in glaucoma patients. Acta Ophthalmol 2013;91:733-7.

17 Michelson G, Scibor M. Intravascular oxygen saturation in retinal vessels in normal subjects and open-angle glaucoma subjects. Acta Ophthalmol Scand 2006;84:289-95.

18 Ito M, Murayama K, Deguchi T, et al. Oxygen saturation levels in the juxta-papillary retina in eyes with glaucoma. Exp Eye Res 2008;86:512-18.

19 Traustason S, Hardarson SH, Gottfredsdottir MS, et al. Dorzolamide-timolo combination and retinal vessel oxygen saturation in patients with glaucoma or ocular hypertension. Br J Ophthalmol 2009;93:1064-7.

20 Siesky B, Harris A, Cantor L, et al. A comparative study of the effects of brinzolamide and dorzolamide on retinal oxygen saturation and ocular microcirculation in patients with primary open-angle glaucoma. $\mathrm{Br}$ I Ophthalmol 2008:92:500-4.

21 Hardarson SH, Gottfredsdottir MS, Halldorsson GH, et al. Glaucoma filtration surgery and retinal oxygen saturation. Invest Ophthalmol Vis Sci 2009:50:5247-50. 


\section{Retinal oxygen metabolism in healthy subjects and glaucoma patients}

Olof Birna Olafsdottir, Evelien Vandewalle, Luis Abegão Pinto, et al.

Br J Ophthalmol 2014 98: 329-333 originally published online January 8,2014

doi: 10.1136/bjophthalmol-2013-303162

Updated information and services can be found at:

http://bjo.bmj.com/content/98/3/329.full.html

These include:

References This article cites 18 articles, 7 of which can be accessed free at: http://bjo.bmj.com/content/98/3/329.full.html\#ref-list-1

Article cited in:

http://bjo.bmj.com/content/98/3/329.full.html\#related-urls

Email alerting

Receive free email alerts when new articles cite this article. Sign up in the box at the top right corner of the online article.

service

Topic

Articles on similar topics can be found in the following collections

Collections

Angle (908 articles)

Glaucoma (893 articles)

Intraocular pressure (905 articles)

Notes

To request permissions go to:

http://group.bmj.com/group/rights-licensing/permissions

To order reprints go to:

http://journals.bmj.com/cgi/reprintform

To subscribe to BMJ go to:

http://group.bmj.com/subscribe/ 